\title{
Predicción de estatura e índice de masa corporal a partir de segmentos corporales. Utilización de las ecuaciones de Gauld en una muestra argentina
} Prediction of height and body mass index based on body segments. Use of Gauld's equations in an Argentine sample

\author{
Dra. Rosario Ramos Mejía , Dra. Silvia Caino ${ }^{a}$, Antropometrista Paula Adamoa, \\ Dra. Virginia Fano y Dra. Diana Kelmansky ${ }^{b}$
}

\section{RESUMEN}

En niños que, por su condición, la estatura no se puede medir o no representa el crecimiento óseo real, puede recurrirse a su cálculo a partir de la medición de segmentos corporales.

Objetivos. 1. Comparar el valor de la estatura observada (EO) y predicha (EP) a partir de segmentos corporales mediante las ecuaciones de Gauld et al. 2. Analizar la aplicabilidad en el cálculo e interpretación del índice de masa corporal (IMC).

Materiales y métodos. Muestra de niños y adolescentes sin alteraciones músculoesqueléticas. Se registró estatura, envergadura, longitud del cúbito, antebrazo, tibia, altura de la pierna, peso y desarrollo puberal. Se calculó el IMC. Se analizaron diferencias y concordancias entre EO y EP mediante Bland-Altman y coeficiente de correlación intraclase. Para el IMC, se calculó el error de predicción absoluto y la concordancia mediante coeficiente kappa. Resultados. Se incluyeron 220 niños y adolescentes entre 6,04 y 19,1 años. El coeficiente de correlación intraclase entre EP y EO fue $>0,9$ en todas las ecuaciones. La EP sobreestimó en promedio la EO en menos de 2,0 cm, excepto al utilizar la medición del cúbito $(2,6 \mathrm{~cm}$ en mujeres y $3,4 \mathrm{~cm}$ en varones). El promedio del error de predicción absoluto para IMC fue $<5 \%$, excepto para el cúbito y el coeficiente kappa fue $>0,7$. Conclusiones. En nuestra muestra, las ecuaciones de Gauld et al. fueron adecuadas para predecir la estatura y calcular el IMC. La mayor diferencia entre EO y EP fue al utilizar la longitud del cúbito. Palabras clave: reproducibilidad de los resultados, predicción, estatura, segmentos corporales, índice de masa corporal.

\section{Correspondencia:}

Dra. Rosario Ramos Mejía: rosariorm@gmail.com

Financiamiento:

Este trabajo fue parcialmente financiado con una Beca de Programa Especial del Hospital Garrahan.

Conflicto de intereses: Ninguno que declarar. http:/ / dx.doi.org/10.5546/aap.2018.e721 Texto completo en inglés:

http:// dx.doi.org/10.5546/aap.2018.eng.e721

Cómo citar: Ramos Mejía R, Caino S, Adamo P, Fano V, et al. Predicción de estatura e índice de masa corporal a partir de segmentos corporales. Utilización de las ecuaciones de Gauld en una muestra argentina. Arch Argent Pediatr 2018;116(6):e721-e729.

\section{GLOSARIO}

CCI: coeficiente de correlación intraclase.

DE: desvío estándar.

ETM: error técnico de medición.

EO: estatura observada.

EP: estatura predicha.

IC: intervalo de confianza.

IMC: índice de masa corporal.

sDE: puntaje del desvío estándar.

\section{INTRODUCCIÓN}

La medición de la estatura es una de las técnicas antropométricas más utilizadas en la práctica médica como indicador del crecimiento, estado nutricional y salud general del niño. Conocer el dato de estatura es necesario para el cálculo de índices e indicadores, tales como el índice de masa corporal (IMC), la superficie corporal, la dosis de fármacos, la interpretación de la presión arterial, la función renal o la función respiratoria.

En toda herramienta para evaluar el crecimiento, se pretende que las mediciones sean lo más exactas y consistentes posible para minimizar el error en la interpretación de los resultados y ulterior toma de decisiones. ${ }^{1,2}$

En ciertas ocasiones, no es posible medir la estatura directamente con el estadiómetro en posición de pie o esta no representa el crecimiento óseo real. Al respecto, se observa, en nuestro Hospital, que, en $24 \%$ de los niños internados, no fue posible medir la estatura por motivos tales como dolor, inmovilización o tubos de drenaje. ${ }^{3}$ Tampoco en niños o adolescentes con enfermedad neurológica, muscular, 
mielomeningocele, contracturas articulares, deformidad espinal o torácica. ${ }^{4,5}$ Estos niños también requieren de la vigilancia del crecimiento y la evaluación del estado nutricional, para lo cual puede recurrirse a la predicción de estatura a partir de segmentos corporales, y el posterior cálculo del IMC.

Gauld et al., publicaron ecuaciones para predecir la estatura a partir de la medición de la envergadura, el cúbito, el antebrazo, la tibia y la pierna con una muestra de niños y adolescentes sanos, caucásicos, de entre 5,3 y 19,5 años. ${ }^{4}$ Estas ecuaciones fueron utilizadas en algunos grupos de pacientes con parálisis cerebral, atrofia muscular espinal y distrofias musculares. ${ }^{6-11} \mathrm{Sin}$ embargo, no se han encontrado, en la literatura, estudios sobre la confiabilidad de la predicción de la estatura mediante estas ecuaciones en otras poblaciones, como tampoco antecedentes de su utilización en nuestro medio, por lo que se ha diseñado este estudio, cuyos objetivos fueron los siguientes: 1. comparar el valor de estatura observada (EO) y predicha (EP) mediante las ecuaciones de Gauld et al. en una muestra de niños y adolescentes argentinos; 2 . analizar la aplicabilidad en el cálculo e interpretación del IMC.

\section{POBLACIÓN Y MÉTODOS}

Se incluyeron niños y adolescentes de entre 6,0 y 19,5 años de edad concurrentes a los consultorios externos del Hospital entre julio de 2015 y julio de 2016. Se incorporaron en forma consecutiva, muestra de conveniencia, hasta alcanzar un mínimo de 100 mujeres y 100 varones (50 con desarrollo puberal en cada grupo y $50 \mathrm{sin}$ él). El tamaño muestral se calculó siguiendo las recomendaciones de Altman para estudios de comparación de métodos. ${ }^{12}$

Se excluyeron niños y adolescentes con baja estatura (menor de -2,0 puntajes del desvío estándar -sDE- para la población argentina), ${ }^{13}$ discapacidad motora o condiciones físicas que impidieran la correcta posición en el estadiómetro, como deformidad torácica o espinal, contracturas articulares, deformidad de los miembros y enfermedades neuromusculares.

La participación fue voluntaria, y se obtuvo el consentimiento informado de los cuidadores y el asentimiento del menor cuando correspondiera.

Un único observador entrenado realizó, en forma replicada y ciega, las mediciones de la estatura, la envergadura, la longitud del cúbito, del antebrazo, de la tibia, la altura de la pierna y el peso. Además, evaluó, por examen físico, el desarrollo puberal y lo clasificó en prepuberal y puberal según los estadios de Tanner. ${ }^{14}$

Un segundo observador entrenado realizó las mismas mediciones antropométricas para calcular el error de medición interobservador de cada técnica, en 10 casos.

Las mediciones de los segmentos corporales se realizaron con un antropómetro Harpenden y la envergadura, con una regla milimetrada diseñada para tal fin, ambos instrumentos con precisión de $1 \mathrm{~mm}$. Las técnicas de medición de segmentos se realizaron siguiendo las recomendaciones de Gauld et al., detalladas en el Anexo. ${ }^{15}$

La medición de la estatura se realizó con un estadiómetro Seca con precisión de $1 \mathrm{~mm}$ y el peso se obtuvo con una balanza de palanca CAM con precisión de 100 g, siguiendo las recomendaciones de la Sociedad Argentina de Pediatría. ${ }^{1}$

Antes de la realización del estudio, se llevó a cabo una prueba piloto con el objetivo de calibrar los instrumentos, poner a punto las técnicas de medición y realizar un entrenamiento en ellas.

El estudio fue aprobado por la Dirección Asociada de Docencia e Investigación y el Comité de Ética del Hospital (Protocolo No 886).

\section{Análisis estadístico}

Paso 1: Replicabilidad y grado de consistencia de cada una de las técnicas antropométricas

El error técnico de medición (ETM) intra- e interobservador para cada una de las técnicas antropométricas se calculó según la fórmula $\left.\mathrm{ETM}=\sqrt{\left[\sum\right.} \mathrm{d}^{2} /(2 \mathrm{n})\right]$, en la que $d$ representa las diferencias entre la primera y la segunda medición, y $n$, el número de casos. Se calculó también el ETM relativo (porcentaje) mediante la fórmula 100 * ETM/x, en la que $x$ es el promedio de las mediciones. ${ }^{16,17}$ Se aceptó un valor límite de ETM relativo $\leq 2 \% .^{16}$

Paso 2: Comparación de la EO y la EP mediante las ecuaciones de Gauld et al.

Se realizó la EP mediante las ecuaciones de Gauld et al., ${ }^{4}$ detalladas en la Tabla 1.

Se dividió la muestra en dos grupos: varones y mujeres. En cada uno, se estratificó en prepuberales y puberales. Para todos los análisis, se consideró el promedio de las mediciones replicadas.

Luego de confirmar la normalidad de las variables con la prueba de KolmogorovSmirnov, se analizaron las diferencias y límites de concordancia entre EO y EP por cada uno de los 
segmentos corporales y entre EO y envergadura observada con el método de Bland-Altman. Este método, basado en la cuantificación del acuerdo entre dos mediciones cuantitativas, permite calcular la media, el DE y el intervalo de confianza con nivel $95 \%$ (IC $95 \%$ ) de la diferencia de medias entre dos métodos. ${ }^{18-20}$

Además, como medida de concordancia, se calculó el coeficiente de correlación intraclase (CCI) entre EO y EP para cada uno de los segmentos corporales. Se consideró concordancia pobre $<0,5$; moderada, entre 0,5 y 0,75 ; buena, entre 0,75 y 0,9 ; excelente, $>0,9 .{ }^{21}$

Paso 3: Comparación del IMC calculado con EO y

EP mediante las ecuaciones de Gauld et al.

Se calculó el IMC - peso (kg)/ talla $^{2}(\mathrm{~m})$ - con EO y EP para cada uno de los segmentos corporales.

Se estimó la media y el DE del error de predicción absoluto (EPA). El EPA se calculó mediante la siguiente fórmula: $\mathrm{EPA}=[100$ * (IMCEO - IMCEP)/IMCEP], en la que IMCEO es el IMC calculado con EO e IMCEP es el IMC calculado con EP por cada uno de los segmentos corporales. $^{22}$

Posteriormente, se clasificaron los valores absolutos del IMC calculados con EO y EP en dos categorías, normal o patológico, teniendo en cuenta los criterios de la Organización Mundial de la Salud (OMS)/National Center for Health Statistics (NCHS) de $2007^{23-25}$ y se evaluó el grado de concordancia mediante el coeficiente de kappaCohen. Se consideró concordancia pobre entre 0,00 y 0,20 ; aceptable, entre 0,21 y 0,40 ; moderada, entre 0,41 y 0,60 ; buena, entre 0,61 y 0,80 ; y muy buena, entre 0,81 y $1,00.12,26$

Para el análisis estadístico, se utilizó el software MedCalc y SPSS.

\section{RESULTADOS}

Se incluyeron 220 niños y adolescentes (114 mujeres), con una mediana de edad de 10,94 (r: de 6,04 a 19,30) años que cumplieron los criterios de inclusión; 105/220 fueron prepuberales. La mediana de estatura fue 0,27 (r: de -1,93 a 1,99) y 0,34 (r: de -2,0 a 2,0) sDE en mujeres y varones, respectivamente.

1. Replicabilidad y grado de consistencia de cada una de las técnicas antropométricas.

El rango del ETM intraobservador fue de 0,13 a $0,17 \mathrm{~cm}$ y el interobservador, de 0,29 a $0,54 \mathrm{~cm}$ para las técnicas de medición de los segmentos corporales. El ETM intra- e interobservador de la estatura fue $0,12 \mathrm{~cm}$ y $0,27 \mathrm{~cm}$, respectivamente.

El ETM relativo intraobservador, que relacionaba el ETM con el promedio de la longitud medida, fue $0,08 \%, 0,15 \%, 0,35 \%, 0,38 \%, 0,51 \%$ y $0,76 \%$ para la estatura, la envergadura, la longitud del antebrazo, la altura de la pierna, la longitud de la tibia y del cúbito, respectivamente. La técnica de medición del cúbito mostró un ETM relativo intraobservador nueve veces mayor respecto al de la estatura. El ETM relativo interobservador de estatura fue $0,17 \%$; de envergadura, $0,26 \%$; del antebrazo, $0,72 \%$; de la tibia, $0,91 \%$; de la pierna, $1,11 \%$ y del cúbito, $1,47 \%$.

2. Comparación de la EO y la EP mediante las ecuaciones de Gauld et al.

La Tabla 2 muestra el promedio y el DE de las diferencias entre EO y EP para cada uno de los segmentos corporales en mujeres y varones prepuberales y puberales. El promedio de las diferencias entre $\mathrm{EO}$ y EP fue menor de $1,2 \mathrm{~cm}$ en mujeres y 1,8 en varones, y fueron mayores en la ecuación con longitud del cúbito y en los grupos con pubertad.

TABLA 1. Ecuaciones propuestas por Gauld et al. para predecir la estatura a partir de segmentos corporales ${ }^{4}$

\begin{tabular}{ll}
\hline Mujeres & \\
\hline EP por envergadura & $\mathrm{EP}=0,619 \times$ envergadura $+1,593 \times$ edad $+36,976$ \\
EP por cúbito & $\mathrm{EP}=4,459 \times$ longitud del cúbito $+1,315 \times$ edad $+31,485$ \\
EP por antebrazo & $\mathrm{EP}=2,908 \times$ longitud del antebrazo $+1,147 \times$ edad $+21,167$ \\
EP por tibia & $\mathrm{EP}=2,771 \times$ longitud de la tibia $+1,457 \times$ edad $+37,748$ \\
EP por pierna & $\mathrm{EP}=2,473 \times$ altura de la pierna $+1,187 \times$ edad $+21,151$ \\
& \\
Varones & $\mathrm{EP}=0,829 \times$ envergadura $+0,721 \times$ edad $+16,258$ \\
EP por envergadura & $\mathrm{EP}=4,605 \times$ longitud del cúbito $+1,308 \times$ edad $+28,003$ \\
EP por cúbito & $\mathrm{EP}=2,904 \times$ longitud del antebrazo $+1,193 \times$ edad $+20,432$ \\
EP por antebrazo & $\mathrm{EP}=2,758 \times$ longitud de la tibia $+1,717 \times$ edad $+36,509$ \\
EP por tibia & $\mathrm{EP}=2,423 \times$ altura de la pierna $+1,327 \times$ edad $+21,818$ \\
EP por pierna &
\end{tabular}

EP: estatura predicha. 
En la Figura 1, parte A, se muestran los gráficos de Bland-Altman entre EO y EP para cada una de las ecuaciones. En el eje vertical, se graficaron las diferencias de estatura entre EO y EP para cada uno de los segmentos corporales y, en el eje horizontal, el promedio de EO y EP. Se observa que la EP sobreestimó significativamente la EO en todas las ecuaciones, a excepción de la ecuación de la envergadura ( $p$ media $=0,052)$ en varones y de la pierna ( $p$ media $=0,145)$ en mujeres. Las diferencias promedio observadas fueron independientes de la estatura del niño, salvo al utilizar la longitud del cúbito $(p=0,001)$, del antebrazo $(p=0,02)$ y la altura de la pierna $(p=0,001)$ en el grupo de varones.

En la parte B de la misma figura, se muestran las diferencias entre $\mathrm{EO}$ y envergadura observada. Las diferencias promedio en mujeres y varones con envergadura observada se modificaron significativamente según el tamaño del niño $(p=0,001)$.
El CCI entre EO y EP fue $>0,9$ en ambos sexos para todas las ecuaciones, y se observó mayor dispersión de los datos en los grupos puberales. 3. Comparación del IMC calculado con EO y EP mediante las ecuaciones de Gauld et al.

El 56,1\% (64/114) de las mujeres y el 55,7 \% (59/106) de los varones mostraron un IMC normal.

En la Tabla 3, se describe el porcentaje del EPA del IMC con EO y EP para cada uno de los segmentos corporales en varones y mujeres prepuberales y puberales. El promedio del porcentaje del EPA del IMC fue $<5 \%$ en ambos sexos, similar para todas las ecuaciones, y fue mayor al utilizar la longitud del cúbito $(5,6 \%)$.

El porcentaje de casos en los que cambió el diagnóstico nutricional fue $<10 \%$, excepto al utilizar la longitud del cúbito en varones con pubertad $(17 \%)$.

Como se observa en la Tabla 4, el coeficiente kappa para las categorías diagnósticas de IMC

TABla 2. Diferencia entre estatura observada y estatura predicha mediante las ecuaciones de Gauld et al.

\begin{tabular}{|c|c|c|c|c|c|}
\hline & $\begin{array}{c}\text { EO-EP de la } \\
\text { envergadura }(\mathrm{cm})\end{array}$ & $\begin{array}{c}\text { EO-EP del } \\
\text { cúbito }(\mathrm{cm})\end{array}$ & $\begin{array}{c}\text { EO-EP del } \\
\text { antebrazo }(\mathrm{cm})\end{array}$ & $\begin{array}{c}\text { EO-EP de } \\
\text { la tibia }(\mathrm{cm})\end{array}$ & $\begin{array}{c}\text { EO-EP de } \\
\text { la pierna }(\mathrm{cm})\end{array}$ \\
\hline & Media (DE) & Media (DE) & Media (DE) & Media (DE) & Media (DE) \\
\hline \multicolumn{6}{|l|}{ Mujeres } \\
\hline Todas $(\mathrm{n}=114)$ & $-1,04(3,28)$ & $-2,62(4,18)$ & $-1,14(3,27)$ & $-0,81(3,37)$ & $0,45(3,27)$ \\
\hline Prepuberales $(n=50)$ & $-0,88(2,40)$ & $-2,01(3,27)$ & $-0,87(2,74)$ & $-0,77(2,54)$ & $0,75(3,00)$ \\
\hline Puberales $(n=64)$ & $-1,18(3,85)$ & $-3,10(4,74)$ & $-1,35(3,65)$ & $-0,84(3,91)$ & $0,21(3,47)$ \\
\hline \multicolumn{6}{|l|}{ Varones } \\
\hline Todos $(\mathrm{n}=106)$ & $-0,52(2,75)$ & $-3,37(3,62)$ & $-1,77(3,05)$ & $-1,33(3,40)$ & $-0,01(3,17)$ \\
\hline Prepuberales $(n=55)$ & $-0,30(2,60)$ & $-2,38(2,90)$ & $-1,26(2,86)$ & $-1,03(2,74)$ & $0,87(2,98)$ \\
\hline Puberales $(n=51)$ & $-0,76(2,91)$ & $-4,43(4,02)$ & $-2,32(3,17)$ & $-1,65(4,00)$ & $-0,96(3,13)$ \\
\hline
\end{tabular}

EO: estatura observada; EP: estatura predicha; DE: desvío estándar.

TABLA 3. Error de predicción absoluto del indice de masa corporal calculado con estatura observada y estatura predicha por las ecuaciones de Gauld

\begin{tabular}{|c|c|c|c|c|c|}
\hline & Envergadura & Cúbito & Antebrazo & Tibia & Pierna \\
\hline \multicolumn{6}{|l|}{ Mujeres } \\
\hline Todas $(\mathrm{n}=114)$ & $3,77(2,96)$ & $5,37(4,54)$ & $3,76(3,04)$ & $3,93(2,70)$ & $3,68(2,73)$ \\
\hline Prepuberales $(n=50)$ & $3,24(2,45)$ & $4,70(3,88)$ & $3,45(2,83)$ & $3,51(2,21)$ & $3,80(2,90)$ \\
\hline Puberales $(n=64)$ & $4,18(3,27)$ & $5,89(4,96)$ & $4,0(3,20)$ & $4,26(3,00)$ & $3,59(2,61)$ \\
\hline \multicolumn{6}{|l|}{$\begin{array}{l}\text { Varones } \\
\% \text { de EPA Media (DE) }\end{array}$} \\
\hline Todos $(n=106)$ & $3,24(2,18)$ & $5,58(3,97)$ & $3,98(2,87)$ & $4,00(3,12)$ & $3,65(2,61)$ \\
\hline Prepuberales $(n=55)$ & $3,26(2,30)$ & $4,73(3,41)$ & $3,85(2,82)$ & $3,59(2,57)$ & $3,89(2,76)$ \\
\hline Puberales $(n=51)$ & $3,22(2,06)$ & $6,49(4,34)$ & $4,11(2,94)$ & $4,45(3,59)$ & $3,40(2,45)$ \\
\hline
\end{tabular}

EPA (error de predicción absoluto) $=[100$ * (IMCEO - IMCEP)/IMCEP].

DE: desvío estándar. IMC: índice de masa corporal. 
Figura 1. PARTE A. Diferencias y límites de concordancia entre estatura observada y estatura predicha por cada una de las ecuaciones utilizando Bland-Altman $(\mathrm{cm})$
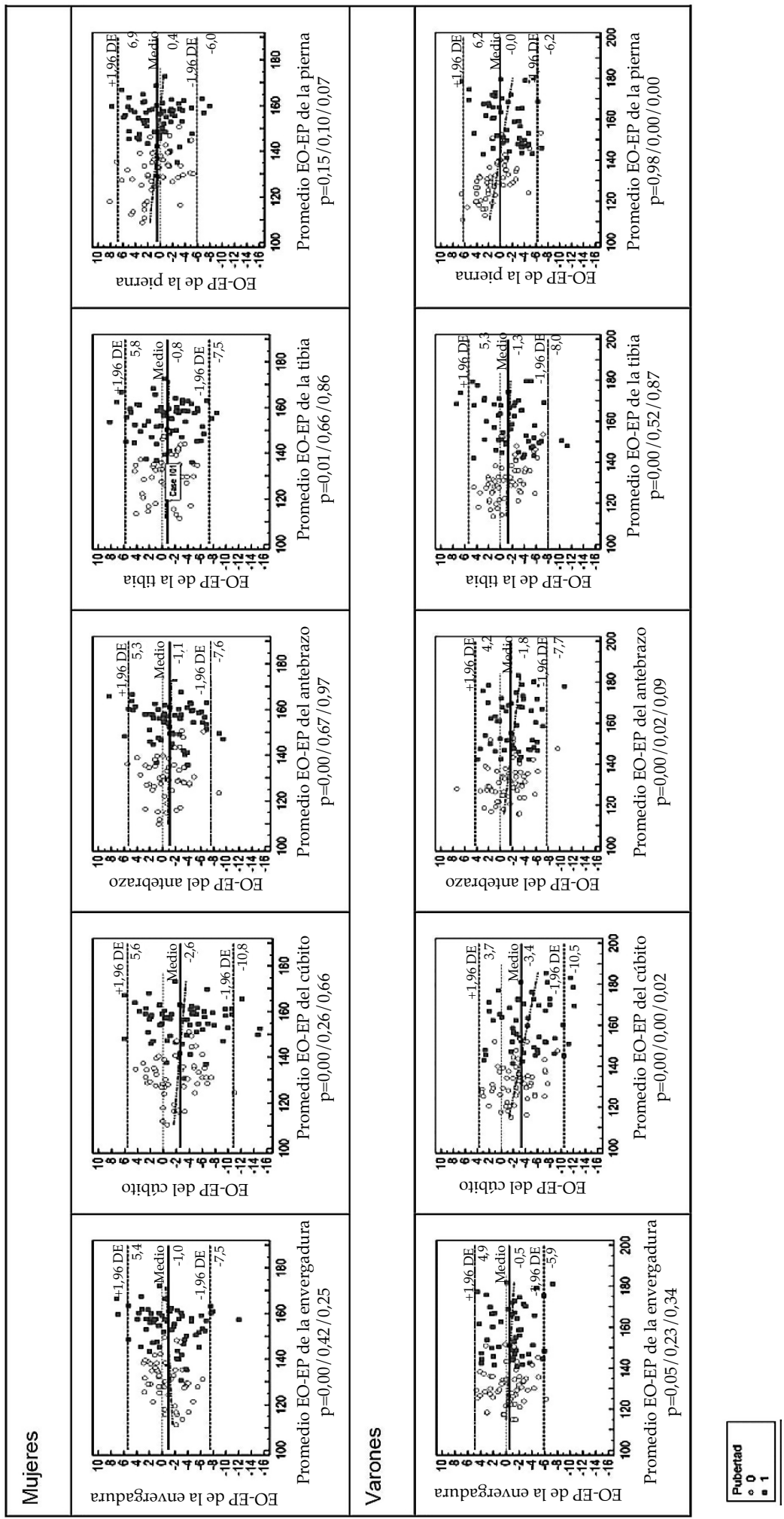

EO: estatura observada; EP: estatura predicha. $\mathrm{p}$ media/ $\mathrm{p}$ pendiente/ $\mathrm{p}$ intercepto.

En el eje $\mathrm{Y}$, se muestran las diferencias entre EO y EP en centímetros y, en el eje X, el promedio de EO y EP en centímetros. 


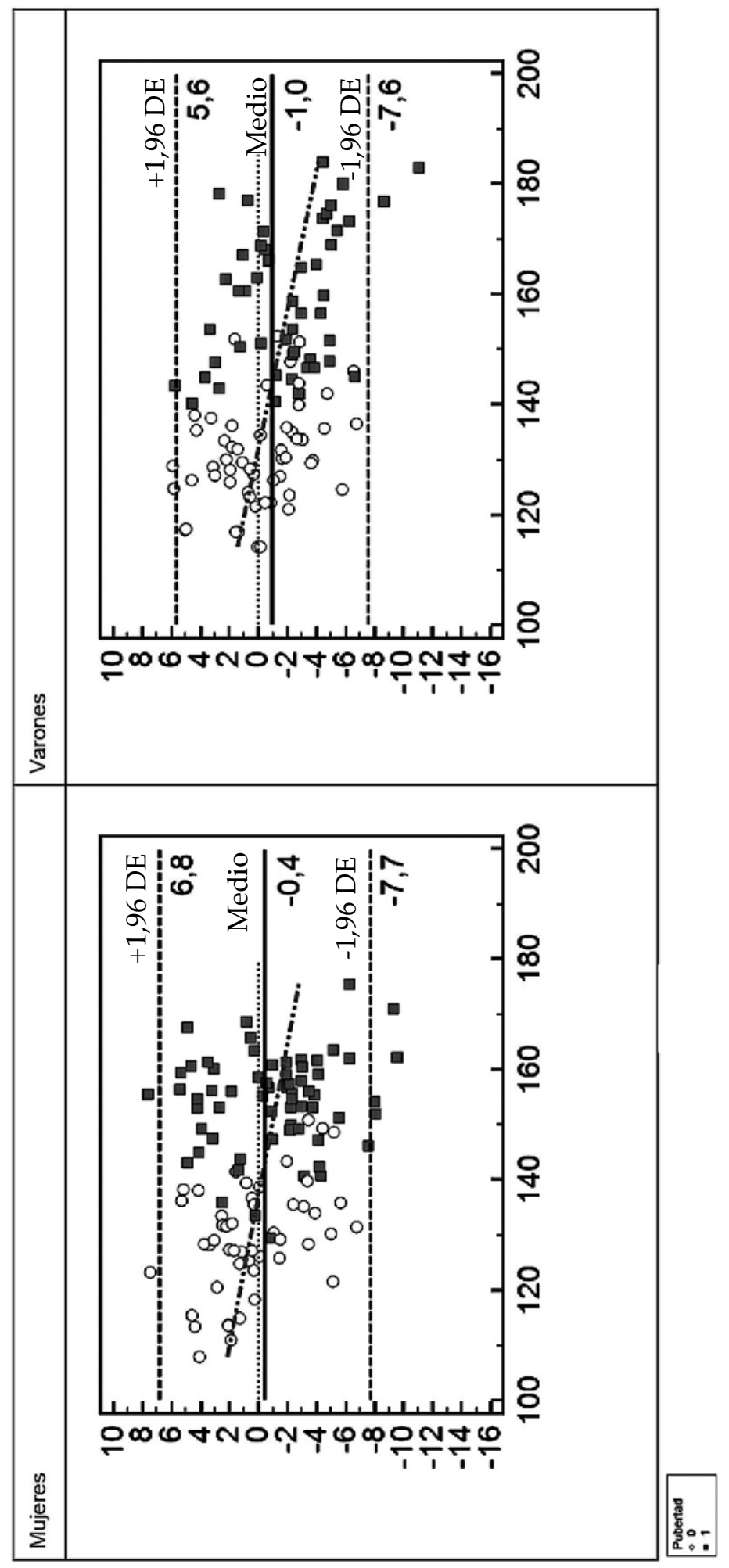

EO: estatura observada. $\mathrm{p}$ media/p pendiente/p intercepto.

En el eje Y, se muestran las diferencias entre EO y en evergadura observada en centímetros y, en el eje X, el promedio de EO y evergadura observada en centímetros. 
fue $\geq 0,70$ para todas las ecuaciones y fue mayor en mujeres prepuberales y varones.

\section{DISCUSIÓN}

En nuestro estudio, se observa una excelente concordancia entre la EO y la EP a partir de los segmentos corporales, mediante las ecuaciones de Gauld et al. La EP sobreestimó en promedio la EO en menos de $2 \mathrm{~cm}$; estos valores fueron mayores en la ecuación con longitud del cúbito y en los grupos con pubertad. Estas diferencias fueron menores que las observadas por Haapala et al., quienes describieron diferencias de hasta $7,3 \mathrm{~cm}$ entre EO y EP en niños con parálisis cerebral. ${ }^{8}$

En la clínica, es frecuente utilizar directamente el valor de la envergadura como indicador de la estatura; ${ }^{27-30}$ sin embargo, se observa, al igual que Golshan et al., que el promedio de las diferencias entre la envergadura observada y la estatura se modificó según el tamaño del niño. ${ }^{31}$ Dado que la predicción de la estatura mediante la ecuación analizada mostró mayor exactitud, se recomienda utilizar la ecuación de Gauld para predecir la estatura a partir de la envergadura.

Los reparos anatómicos de las técnicas de medición de segmentos corporales fueron fáciles de identificar y el ETM relativo se encontró dentro de los límites aceptados para la evaluación clínica del crecimiento. ${ }^{1,16}$ Sin embargo, a diferencia de lo propuesto por Gauld et al., se encontró cierta dificultad en la localización de la apófisis estiloides del cúbito en algunos niños, en especial, en aquellos con sobrepeso. Esta dificultad se reflejó en el ETM relativo intra- e interobservador mayor que el obtenido con el resto de los segmentos y con la medición de la estatura.

Se encontró una fuerte concordancia (coeficiente kappa $\geq 0,7$ ) entre las categorías diagnósticas del IMC calculado con EO y EP por las ecuaciones de Gauld et al. Sin embargo, y teniendo en cuenta que un porcentaje de casos cambió el diagnóstico nutricional entre el IMC calculado con EO y EP, se sugiere analizar la evolución en el tiempo de este indicador más que realizar un corte transversal.

La menor concordancia entre las categorías diagnósticas de IMC al utilizar la ecuación con longitud del cúbito, al igual que la mayor diferencia entre EO y EP mediante este segmento, podría estar asociada a la mayor dificultad en la técnica de medición reflejada por el mayor ETM relativo respecto a los otros segmentos. Por lo antes mencionado, se sugiere, además de un período previo de entrenamiento para minimizar el error de medición, utilizar el promedio de, al menos, dos mediciones replicadas y emplear siempre el mismo segmento corporal en el seguimiento longitudinal del niño y/o adolescente.

Se conoce que las proporciones corporales cambian durante la pubertad y, además, la edad de inicio de la pubertad varía entre los niños. Al respecto, se observa que las diferencias entre EO y EP fueron mayores; el ICC y el coeficiente kappa fueron menores en el grupo con pubertad. Sobre la base de estos hallazgos, se propone que, además de la edad, el sexo y el segmento corporal, habría que analizar la inclusión del desarrollo puberal como factor de corrección en futuras ecuaciones con el objetivo de mejorar la predicción de la estatura.

El presente trabajo es el primero que analiza la confiabilidad de las ecuaciones de Gauld et al. para predecir la estatura a partir de la medición de segmentos corporales, y su utilización en el cálculo de IMC, en una muestra de niños y adolescentes argentinos sin discapacidad motora y con estatura normal para la población de referencia.

Dado que estas ecuaciones han sido propuestas para niños y adultos con distintas condiciones neuromusculares, tales como parálisis cerebral, atrofia muscular espinal y distrofinopatías, ${ }^{6-11}$

TABLA 4. Concordancia entre categorías diagnósticas de índice de masa corporal (normal, patológico) calculado con estatura observada y estatura predicha. Coeficiente kappa

\begin{tabular}{|c|c|c|c|c|c|}
\hline & Envergadura & Cúbito & Antebrazo & Tibia & Pierna \\
\hline \multicolumn{6}{|l|}{ Mujeres } \\
\hline Todas $(\mathrm{n}=114)$ & 0,77 & 0,70 & 0,77 & 0,79 & 0,75 \\
\hline Prepuberales $(n=50)$ & 0,88 & 0,84 & 0,88 & 0,88 & 0,88 \\
\hline Puberales $(n=64)$ & 0,68 & 0,59 & 0,69 & 0,72 & 0,65 \\
\hline \multicolumn{6}{|l|}{ Varones } \\
\hline Todos $(n=106)$ & 0,92 & 0,81 & 0,89 & 0,85 & 0,91 \\
\hline Prepuberales $(n=55)$ & 0,93 & 0,71 & 0,85 & 0,82 & 0,82 \\
\hline Puberales $(n=51)$ & 0,92 & 0,92 & 0,92 & 0,88 & 1,00 \\
\hline
\end{tabular}


sería importante evaluar, en un futuro, su confiabilidad, así como seleccionar el segmento corporal que mejor predice la estatura, en esta población.

\section{CONCLUSIONES}

Basados en nuestros resultados, cuando por diferentes razones no se pueda medir la estatura, se considera que las ecuaciones de Gauld et al., son adecuadas para su predicción, así como para calcular el IMC. La mayor diferencia entre EO y EP se observó al utilizar la longitud del cúbito.

\section{REFERENCIAS}

1. Comité Nacional de Crecimiento y Desarrollo. Sociedad Argentina de Pediatría. Guía para la evaluación del crecimiento físico. 2013. [Acceso: 8 de junio de 2018]. Disponible en: http://www.sap.org.ar/docs/ publicaciones/libro_verde_sap_2013.pdf.

2. World Health Organization Multicentre Growth Reference Study Group. WHO Child Growth Standards: Length/ height-for-age, weight-for-age, weight-for-length, weightfor-height and body mass index-for-age: Methods and development. Geneva: World Health Organization, 2006. [Acceso: 4 de septiembre de 2017]. Disponible en: http: / / www.who.int/childgrowth/standards/technical_report/ en/.

3. Spirito MF, CainoS, Vezzani C, et al. Uso dela antropometría para la evaluación nutricional en pacientes internados en un hospital pediátrico de alta complejidad: su aplicabilidad y limitaciones. Med Infant 2017; 24(1):8-13.

4. Gauld LM, Kappers J, Carlin JB, Robertson CF. Height prediction from ulna length. Dev Med Child Neurol 2004; 46(7):475-80.

5. Forman MR, Zhu Y, Hernández LM, et al. Arm span and ulnar length are reliable and accurate estimates of recumbent length and height in a multiethnic population of infants and children under 6 years of age. J Nutr 2014; 144(9):1480-7.

6. Bönnemann CG, Rutkowski A, Mercuri E, et al. 173rd ENMC international workshop: Congenital muscular dystrophy outcome measures 5-7 March 2010, Naarden, The Netherlands. Neuromuscul Disord 2011; 21(7):513-22.

7. McDonald CM, Henricson EK, Abresch RT, et al. The cooperative international neuromuscular research group duchennenatural history study-a longitudinal investigation in the era of glucocorticoid therapy: Design of protocol and the methods used. Muscle Nerve 2013; 48(1):32-54.

8. Haapala H, Peterson MD, Daunter A, Hurvitz EA. Agreement between actual height and estimated height using segmental limb lengths for individuals with cerebral palsy. Am J Phys Med Rehabil 2015; 94(7):539-46.

9. Kaufmann P,McDermottMP, Darras BT, etal. Observational Study of Spinal Muscular Atrophy Type 2 and 3: functional Outcomes Over 1 Year. Arch Neurol 2011; 68(6):779-86.

10. Meier T, Rummey C, Leinonen M, et al. Characterization of pulmonary function in 10-18 year old patients with Duchenne muscular dystrophy. Neuromuscul Disord 2017; 27(4):307-14.
11. Wood CL, Straub V, Guglieri M, et al. Short stature and pubertal delay in Duchenne muscular dystrophy. Arch Dis Child 2016; 101(1):101-6.

12. Altman DG. Methods comparison studies. In: Altman DG. Practical Statistics for Medical Research. Londres: Chapman \& May; 1991.p.396-409.

13. Lejarraga $\mathrm{H}$, del Pino M, Fano V, et al. Referencias de peso y estatura desde el nacimiento hasta la madurez para niñas y niños argentinos: Incorporación de datos de la OMS de 0 a 2 años, recálculo de percentilos para obtención de valores LMS. Arch Argent Pediatr 2009; 107(2):126-33.

14. Tanner JM. Growth at adolescence. 2nd ed. Oxford: Blackwell, 1962.

15. Gauld LM, Kappers J, Carlin JB, Robertson CF. Prediction of Childhood Pulmonary Function Using Ulna Length. Am J Respir Crit Care Med 2003; 168(7):804-9.

16. Norton K, Olds T (edt). Anthropometrica: A Textbook of Body Measurement for Sports and Health Courses. Sidney: UNSW Press, 1996.

17. Cameron N. The Methods of Auxological Anthropometry. En: Falkner F, Tanner JM. Human growth: A comprehensive treatise. 2nd ed. Londres: Plenum Press, 1986.p.3-43.

18. Altman DG, Bland JM. Measurement in Medicine: The Analysis of Method Comparison Studies. J R Stat Soc Series D Stat 1983; 32(3):307-17.

19. Altman DG, Bland JM. Statistical methods for assessing agreement between two methods of clinical measurement. Lancet 1986; 8(8476):307-10.

20. Giavarina D. Understanding Bland Altman analysis. Biochemia Med (Zagreb). 2015; 25(2):141-51.

21. Koo TK, Li MY. A Guideline of Selecting and Reporting Intraclass Correlation Coefficients for Reliability Research. J Chiropr Med 2016; 15(2):155-63.

22. Smith RJ. Rethinking Allometry. J Theor Biol. 1980;87(1):97111.

23. World Health Organization. Growth reference data for 5-19 years. [Acceso: 4 de septiembre de 2017]. Disponible en: http: / / www.who.int/growthref.

24. De Onis M, Lobstein T. Defining obesity risk status in the general childhood population: Which cut-offs should we use? Int J Pediatr Obes 2010; 5(6):458-60.

25. Cole TJ, Flegal KM, Nicholls D, Jackson AA. Body mass index cut offs to define thinness in children and adolescents: international survey. BMJ 2007; 335(7612):194.

26. Landis JR, Koch GG. The Measurement of Observer Agreement for Categorical Data. Biometrics 1977;33(1):15974.

27. Aggarwal AN, Gupta D, Ezekiel LM, Jindal SK. Statistical estimation of height from arm Span in north indian subjects. Indian J Physiol Pharmacol 2000; 44(3):329-34.

28. Datta BanikS. Arm span as a proxy measure for height and estimation of nutritional Status: a study among dhimals of darjeeling in West Bengal India. Ann Hum Biol 2011; 38(6):728-35.

29. Zverev Y, Chisi J. Estimating height from arm span measurement in Malawian children. Coll Antropol 2005; 29(2):469-73.

30. Yousafzai AK, Filteau SM, WirzSL, Cole TJ. Comparison of armspan, arm length and tibia length as predictors of actual height of disabled and nondisabled children in Dharavi, Mumbai, India. Eur J Clin Nutr 2003; 57(10):1230-4.

31. Golshan M, Amra B, Hoghoghi MA. Is arm span an accurate measure of height to predict pulmonary function parameters? Monaldi Arch Chest Dis 2003; 59(3):189-92. 


\section{ANEXO}

\section{TÉCNICAS DE MEDICIÓN DE SEGMENTOS CORPORALES}

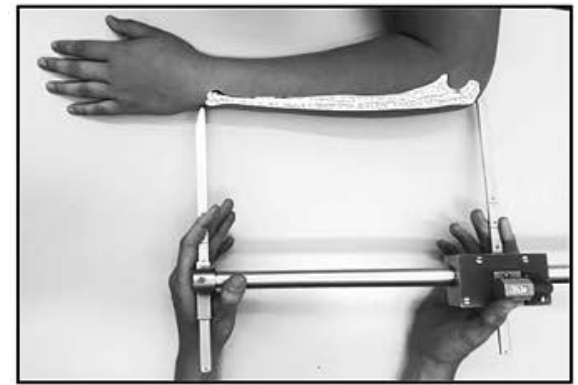

Longitud de cúbito

La longitud del cúbito se midió entre la apófisisestiloides y el extremo distal del codo. El niño se colocó sentado, conel codo izquierdo flexionado en 90 a $110^{\circ}$, el antebrazo apoyado sobre una mesa, la palma de la mano hacia abajo y los dedos juntos extendidos.

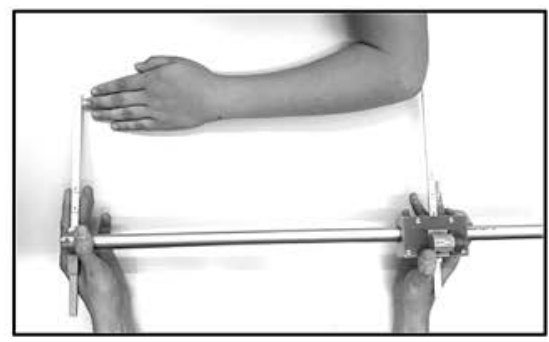

Longitud de antebrazo

La medición del antebrazo se realizó entre el extremos distal del dedo medio izquierdo y el codo. El niño se colocó en la misma posición que para medir la longitud del cúbito.

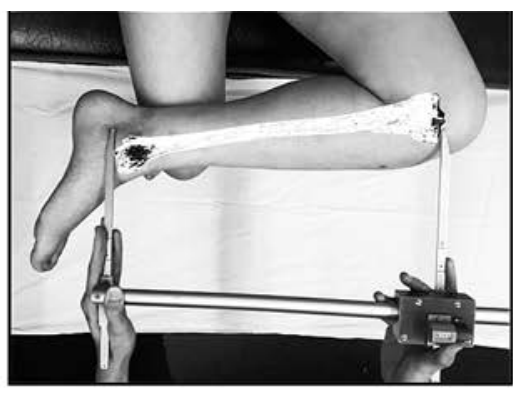

\section{Longitud de tibia}

La longitud de la tibia se realizó entre el platillo tibial interno y el punto más distal del maléolo interno. El niño se colocó en posición sentada con la pierna izquierda cruzada sobre la derecha y la rodilla en flexión.

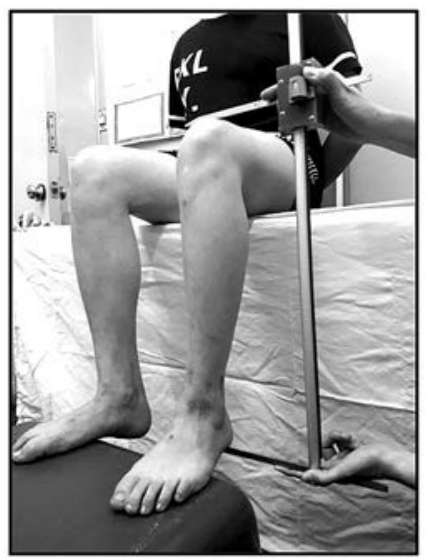

Altura de pierna

La altura de la pierna izquierda se midió entre la parte superior de la rodilla y el talón. El niño se colocó en posición sentada, con la rodilla flexionada en $90^{\circ}$ a la misma altura que la cadera y el pie apoyado sobre una superficie plana en $90^{\circ}$ respecto al eje longitudinal de la pierna.

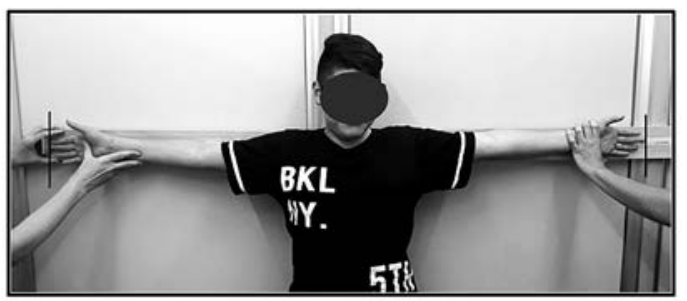

Envergadura

La envergadura esla distancia entreel punto más extremo del dedo medio de una mano y su homólogo de la otra mano. La medición se realizó en posición de extensión horizontal máxima de los brazos, perpendiculares al eje axial, con las palmas de las manos hacia adelante.

Adaptado de: Gauld L.M., Kappers J., Carlin J.B., Robertson C.F. Prediction of Childhood Pulmonary Function Using Ulna Length. American Journal Of Respiratory And Critical Care Medicine, 2003; 168: 804-809 